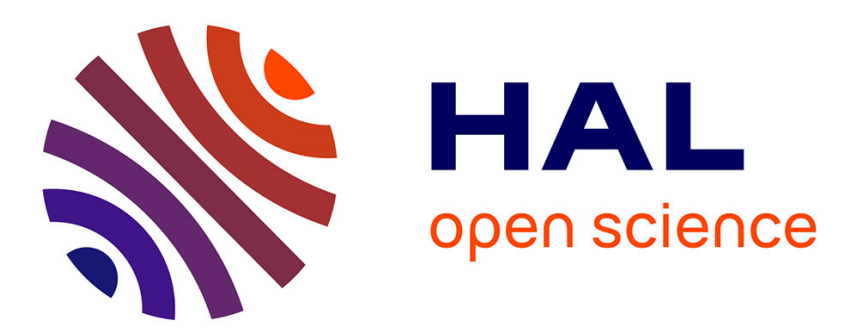

\title{
Abrikosov vortex pinning on a cylindrical cavity inside the vortex core: formation of a bound state and depinning
}

\author{
A. A. Bespalov, A. S. Mel'Nikov
}

\section{- To cite this version:}

A. A. Bespalov, A. S. Mel'Nikov. Abrikosov vortex pinning on a cylindrical cavity inside the vortex core: formation of a bound state and depinning. Superconductor Science and Technology, 2013, 26 (8), pp.085014. 10.1088/0953-2048/26/8/085014 . hal-00858338

\section{HAL Id: hal-00858338 \\ https://hal.science/hal-00858338}

Submitted on 8 Mar 2018

HAL is a multi-disciplinary open access archive for the deposit and dissemination of scientific research documents, whether they are published or not. The documents may come from teaching and research institutions in France or abroad, or from public or private research centers.
L'archive ouverte pluridisciplinaire HAL, est destinée au dépôt et à la diffusion de documents scientifiques de niveau recherche, publiés ou non, émanant des établissements d'enseignement et de recherche français ou étrangers, des laboratoires publics ou privés.

\section{(1)(1) $\$(0)$}

Distributed under a Creative Commons Attribution - NonCommercial - ShareAlikel 4.0 
Abrikosov vortex pinning on a cylindrical cavity inside the vortex core: formation of a bound state and depinning

This article has been downloaded from IOPscience. Please scroll down to see the full text article.

2013 Supercond. Sci. Technol. 26085014

(http://iopscience.iop.org/0953-2048/26/8/085014)

View the table of contents for this issue, or go to the journal homepage for more

Download details:

IP Address: 147.210.24.83

The article was downloaded on 04/09/2013 at 10:26

Please note that terms and conditions apply. 


\title{
Abrikosov vortex pinning on a cylindrical cavity inside the vortex core: formation of a bound state and depinning
}

\author{
A A Bespalov ${ }^{1,2}$ and A S Mel'nikov ${ }^{1}$ \\ ${ }^{1}$ Institute for Physics of Microstructures, Russian Academy of Sciences, GSP-105, 603950, \\ Nizhny Novgorod, Russia \\ ${ }^{2}$ Université Bordeaux, LOMA, UMR 5798, F-33600 Talence, France \\ E-mail: bespalovaa@gmail.com
}

\begin{abstract}
Within the Ginzburg-Landau theory we consider Abrikosov vortex pinning on a columnar defect with the characteristic size of the cross-section much smaller than the coherence length. We present a simple analytical approach to the problem of pinning potential calculation suitable for cases of the defect situated inside as well as outside the vortex core. We determine the depinning current for a cavity in the form of a circular cylinder and the pinning potential for a defect with a general elliptical cross-section. For a range of applied currents below the depinning threshold we predict the possibility to observe a stable bound state where the vortex center is located outside the cavity.
\end{abstract}

\section{Introduction}

The possibilities for practical applications of type-II superconductors depend crucially on the quality of vortex pinning structures which can be embedded into these materials. The implantation of artificial columnar defects into superconductors may lead to a drastic increase of the maximal non-dissipative (critical) current, if the vortex lines are aligned along the defects (see [1-3] and references therein). Nowadays, various techniques exist allowing one to create disordered arrays of such defects [1, 2], as well as regular defect lattices [4]. Due to the technological importance of enhancing the critical current, this problem continues to attract the interest of experimental groups [3].

The theoretical investigation of collective pinning and creep of the vortex lattice is known to be a quite complicated task. An extensive review of existing theories, which account for vortex-defect as well as vortex-vortex interactions, can be found in [5]. In order to deduce results which can be quantitatively compared with experimental data, these theories require the individual vortex pinning potential as input. It is known [6] that the efficiency of a pinning array depends not only on the depth of the potential well for a vortex, but also on the shape of the well. Thus, a detailed investigation of single vortex-single defect interactions provides the basis for considerations of many-vortex systems.

The problem of single-vortex pinning is generally formulated as follows. The system (see figures 1(a) and (b)) contains a vortex and a defect, which is typically assumed to be of cylindrical shape. The task is to determine the vortex energy as a function of its position, and the transport current density, $\boldsymbol{j}_{\text {tr }}$, which is sufficient to detach the vortex from the defect. This current density is called the depinning current density.

The first theoretical study of individual vortex pinning on a cylindrical defect was carried out by Mkrtchyan and Schmidt [7]. In their paper the London equation was solved exactly for a vortex interacting with a cavity in the form of a circular cylinder. The pinning force was analyzed in detail for the cavity radius $a$ satisfying the condition $\xi \ll a \ll \lambda$, where $\xi$ is the coherence length and $\lambda$ is the London length. Later [8, 9], this analysis was extended to the case of larger cavities. Buzdin and Feinberg [10] pointed out that London screening can be neglected in a large range of fields in extreme type-II superconductors. This observation allowed them to establish an electrostatic analogy and to simplify considerably the solution for a vortex interacting with a circular cavity: it was demonstrated that the full magnetic field can be presented as the sum of the vortex self-field and the field of image 
(a)

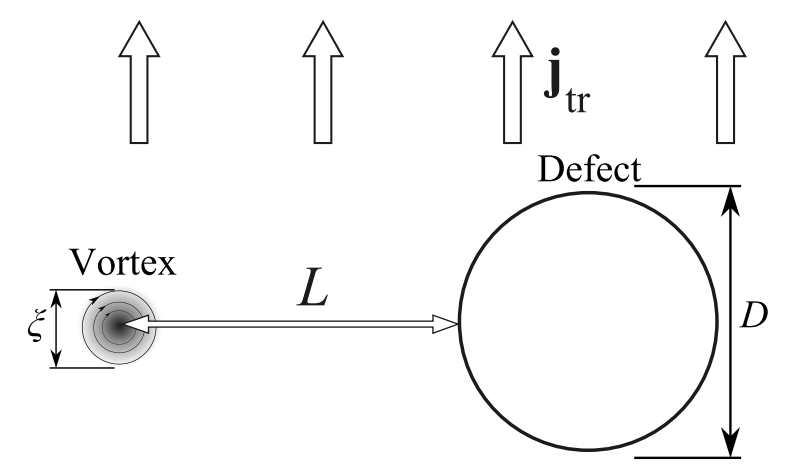

(b)

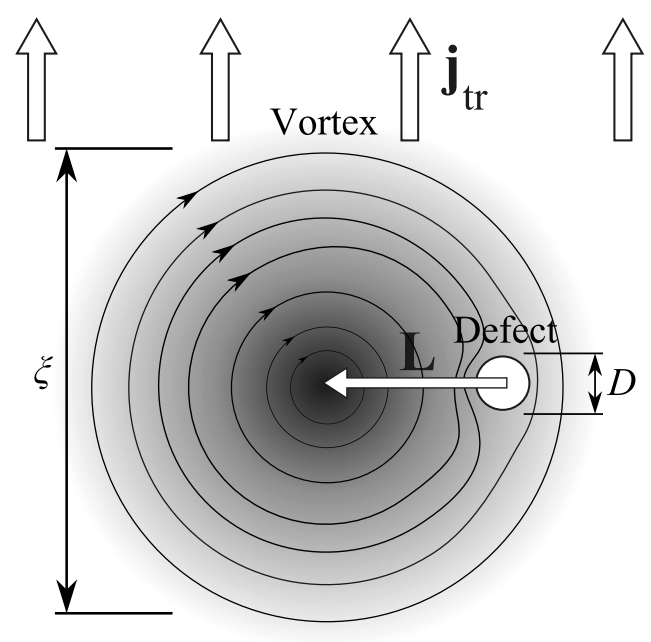

Figure 1. The cross-section of the system. (a) The case when the London theory can be applied: the defect size $D$ and vortex-defect distance $L$ are large as compared to the coherence length. (b) The case studied in this paper: the defect is small $(D \ll \xi)$ and may be situated inside the vortex core. $\boldsymbol{j}_{\mathrm{tr}}$ is the transport current density.

vortices, situated inside the cavity. Also, using the conformal transformation technique, pinning potentials for more tricky columnar cavities have been derived $[11,12]$. However, in the calculation of the pinning potential for non-circular defects, only the field of the image vortices has been transformed, while the modification of the self-field of the real vortex has not been taken into account.

We have to note that the London theory is valid in a limited range of parameters: the characteristic defect size $D$ and vortex-defect distance $L$ must satisfy the conditions $D \gg \xi$ and $L \gg \xi$ (see figure 1(a)). In order to analyze smaller defects, a more complex approach is required. For temperatures close to the superconducting critical temperature, the Ginzburg-Landau (GL) approximation is a natural choice. It has been applied for the analysis of vortex pinning on columnar defects of different shape and nature [5, 13-16] (also, recently the GL equation has been used to study the interaction of vortices with 1D pinning inclusions [17]). Due to the nonlinearity of the GL equation, its analytical solution presents a theoretical challenge, especially in the noncentrosymmetric case. For that reason this equation has been mainly analyzed numerically. Maurer et al [13] calculated the pinning energy for a vortex centered on a circular insulating or metallic inclusion. Later [14], the depinning current for defects with radii of $0.25 \xi$ and larger was determined by solving the GL equation in two dimensions. Priour and Fertig [15] analyzed vortex interactions with a cylindrical hole with a square cross-section. In [16] the critical current for a vortex lattice pinned on a set of defects with reduced critical temperature was determined using numerical simulations and a variational procedure. A simple analytical estimate for the single-vortex pinning energy is given in the review by Blatter et al [5]. The authors applied the variational principle, taking into account the suppression of the order parameter inside the defect and ignoring the local vortex distortion (the GL boundary condition is not satisfied on the defect border). Thus, exact analytical solutions of the GL equation have been lacking so far.

In this paper, within the GL theory we consider the interaction of a vortex with a small cylindrical cavity or insulating inclusion with the characteristic size of the cross-section $\xi_{0} \ll D \ll \xi$, where $\xi_{0}$ is the zero-temperature coherence length (in the case $D \ll \xi_{0}$ the correct description can be obtained only on the basis of a microscopic theory [18-20]). We present the exact pinning potentials in terms of the unperturbed vortex order parameter for a circular and elliptical defect. For the circular cavity the depinning current is also determined. For the treatment of a small defect with arbitrary cross-section we propose an electrostatic analogy, which serves as a counterpart to the above-mentioned analogy between the London theory (valid for large defects, $D \gg \xi$ ) and electrostatics. Unlike the London theory electrostatics, our approach allows one to treat rigorously the cases of the defect situated outside as well as inside the vortex core (see figure 1(b)). Finally, using the conformal transformation method developed in [12], we derive the pinning potential for an elliptical cavity within the London approximation, taking into account the modification of both the vortex self-field and the image field.

\section{Vortex pinning within the Ginzburg-Landau theory}

\subsection{Basic equations}

We consider an Abrikosov vortex interacting with a small cylindrical cavity, or insulating inclusion: the characteristic size $D$ of the defect is much smaller than the coherence length $\xi$ (see figure 1(b)). Since vortex pinning is most efficient for the vortex axis parallel to the defect generatrix, we will focus on this particular case. Our goal is to determine the pinning potential and the depinning current. In order to calculate the latter, we introduce a transport current with the density $\boldsymbol{j}_{\text {tr }}$ flowing in the direction perpendicular to the vortex axis. 
The equilibrium state of the system may be analyzed using the GL equation for the order parameter $\psi=|\psi| \mathrm{e}^{\mathrm{i} \theta}$ :

$$
-\xi^{2} \nabla^{2} \psi-\psi+n_{0}^{-1}|\psi|^{2} \psi=0,
$$

where $n_{0}$ is the concentration of Cooper pairs in the bulk. The GL parameter $\kappa=\lambda / \xi$ is assumed to be large, so the vector potential can be neglected [21]. We put the origin of coordinates inside the insulating defect, or cavity, and the $z$-axis along the vortex axis, so that the order parameter does not depend on $z$.

Equation (1) is supplemented by two boundary conditions, specifying the normal derivative of $\psi$ at the insulating defect border and the transport current density far from the vortex core:

$$
\begin{aligned}
\left.\boldsymbol{n} \nabla \psi\right|_{\partial S} & =0, \\
\left.\frac{2 e \hbar|\psi|^{2} \nabla \theta}{m}\right|_{\rho \rightarrow \infty} & =\boldsymbol{j}_{\mathrm{tr}} .
\end{aligned}
$$

Here $S$ is the defect cross-section, $\partial S$ denotes the border of $S, \boldsymbol{n}$ is the outward unit normal to $\partial S, e$ is the electron charge, and $m$ is the Cooper pair mass.

Since our system must contain one vortex, an additional condition for the order parameter phase arises:

$$
\oint \nabla \theta \mathrm{d} l=2 \pi
$$

where integration is performed over a sufficiently large contour surrounding the defect.

We expect that for some current $j_{\mathrm{d}}$ equations (1)-(4) can be solved when $j_{\mathrm{tr}}<j_{\mathrm{d}}$, and no solution exists when $j_{\mathrm{tr}}>j_{\mathrm{d}}$. Then it is natural to consider $j_{\mathrm{d}}$ as a depinning current.

We will solve equations (1)-(4) in the case $j_{\mathrm{tr}}<j_{\mathrm{d}}$. If the transport current is much smaller than the depairing current, the order parameter has the following asymptotics at infinity:

$$
\psi \approx \sqrt{n_{0}} \mathrm{e}^{\mathrm{i} \varphi+\mathrm{i} q \rho},
$$

where $\varphi$ is the polar angle measured from an axis passing through the center of the vortex ( $\varphi$ will be explicitly defined below), and $\boldsymbol{q}=m \boldsymbol{j}_{\mathrm{tr}} / 2 e \hbar n_{0}$. This asymptotics can be derived from (1), (3) and (4) if one expands $\psi$ in powers of $\rho^{-1}$ and neglects terms proportional to $j_{\mathrm{tr}}^{2}$. Now we make some assumptions concerning the behavior of the order parameter in the vicinity of the defect.

(A) The order parameter phase reaches its asymptotic behavior at sufficiently small distances from the origin: $\theta \approx \varphi+\boldsymbol{q} \rho$ when $\rho \geq R$, where $R$ is some radius in the range $\xi \ll R \ll q^{-1}, \lambda$.

(B) The vortex is weakly distorted by a small defect and a small current. This means that the solution of equations (1)-(4) can be presented in the form $\psi=\psi_{0}+\psi_{1}$, where $\psi_{0}$ corresponds to an unperturbed vortex shifted from the origin by a vector $\boldsymbol{L}$ (see figure $1(\mathrm{~b})$ ), and $\psi_{1}$ is a small perturbation: $\left|\psi_{1}(\rho)\right| \ll \sqrt{n_{0}}$ when $\rho<R$.

The assumption (B) is justified by the fact that the unperturbed vortex corresponds to a local minimum of the free energy, so large distortions are not energetically favorable. Both statements (A) and (B) can be verified by numerical calculations. In terms of the vector $\boldsymbol{L}$, the polar angle is defined as

$$
\tan \varphi=\frac{y-L_{y}}{x-L_{x}}
$$

Let us write down the equations for the function $\psi_{1}$. If we linearize equation (1) we obtain

$$
-\xi^{2} \nabla^{2} \psi_{1}-\psi_{1}+2 n_{0}^{-1}\left|\psi_{0}\right|^{2} \psi_{1}+n_{0}^{-1} \psi_{0}^{2} \psi_{1}^{*}=0 .
$$

Far from the vortex core, when the characteristic scale of the order parameter is much larger than $\xi$, we may obtain from the GL equation

$$
\psi \approx \sqrt{n_{0}}\left(1-\frac{\xi^{2}}{2}(\nabla \theta)^{2}\right) \mathrm{e}^{\mathrm{i} \theta} .
$$

Hence, according to the statement (A), the perturbation $\psi_{1}=$ $\psi-\psi_{0}$ of the order parameter is given by

$$
\psi_{1}(\boldsymbol{\rho})=\sqrt{n_{0}} \mathrm{ie}^{\mathrm{i} \varphi}(\boldsymbol{q} \boldsymbol{\rho})+\mathrm{O}\left(\frac{\sqrt{n_{0}} \xi^{2} q}{\rho}\right), \quad \rho \sim R .
$$

The boundary condition at the defect border for $\psi_{1}$ follows from (2):

$$
\left.\left(\nabla \psi_{1}+\nabla \psi_{0}\right) \boldsymbol{n}\right|_{\partial S}=0 .
$$

Thus, equations (6)-(8) are to be solved. Here, for clarity, we would like to stress that the boundary condition (8) accounts for both the variations of the order parameter modulus and phase. It is reduced to the London theory boundary condition, $\nabla \theta \boldsymbol{n}=0$, only if the vortex is far from the defect, i.e. $L \gg \xi$.

\subsection{Variational derivation of the pinning potential}

In this section, for the reader's convenience, we present a relatively simple, but not rigorous derivation of the pinning potential. A more detailed and careful analysis is given in section 2.3 .

We will determine the free energy $F$ per unit length of a vortex shifted from the origin by a vector $\boldsymbol{L}$ :

$$
F=\frac{H_{\mathrm{c}}^{2}}{4 \pi n_{0}} \int_{\boldsymbol{\rho} \notin S}\left(\xi^{2}|\nabla \psi|^{2}-|\psi|^{2}+n_{0}^{-1} \frac{|\psi|^{4}}{2}\right) \mathrm{d}^{2} \boldsymbol{\rho},
$$

where $H_{\mathrm{c}}$ is the thermodynamic critical field satisfying the relation

$$
\frac{H_{\mathrm{c}}^{2}}{8 \pi}=\frac{\hbar^{2} n_{0}}{4 m \xi^{2}} .
$$

In the zero-order approximation $F$ equals the free energy of an unperturbed vortex:

$$
F_{0}=\frac{H_{\mathrm{c}}^{2}}{4 \pi n_{0}} \int\left(\xi^{2}\left|\nabla \psi_{0}\right|^{2}-\left|\psi_{0}\right|^{2}+n_{0}^{-1} \frac{\left|\psi_{0}\right|^{4}}{2}\right) \mathrm{d}^{2} \boldsymbol{\rho} .
$$

The pinning potential equals the difference between the exact free energy $F$ and $F_{0}: U_{\mathrm{p}}(\boldsymbol{L})=F(\boldsymbol{L})-F_{0}$. This difference consists of two terms $-\Delta F_{1}$ and $\Delta F_{2}$. The first term is 
connected with the suppression of the order parameter inside the defect:

$$
\Delta F_{1} \approx-\left.S \frac{H_{\mathrm{c}}^{2}}{4 \pi n_{0}}\left(\xi^{2}\left|\nabla \psi_{0}\right|^{2}-\left|\psi_{0}\right|^{2}+n_{0}^{-1} \frac{\left|\psi_{0}\right|^{4}}{2}\right)\right|_{\rho=0} .
$$

Note that this expression is valid only for very small defects with the characteristic size of the cross-section $D \ll \xi$. The second term is connected with the distortion of the order parameter outside the defect:

$$
\begin{aligned}
\Delta F_{2}= & \frac{H_{\mathrm{c}}^{2}}{4 \pi n_{0}} \int_{\boldsymbol{\rho} \notin S}\left(\xi^{2}|\nabla \psi|^{2}-|\psi|^{2}+n_{0}^{-1} \frac{|\psi|^{4}}{2}\right) \mathrm{d}^{2} \boldsymbol{\rho} \\
& -\frac{H_{\mathrm{c}}^{2}}{4 \pi n_{0}} \int_{\boldsymbol{\rho} \notin S}\left(\xi^{2}\left|\nabla \psi_{0}\right|^{2}-\left|\psi_{0}\right|^{2}+n_{0}^{-1} \frac{\left|\psi_{0}\right|^{4}}{2}\right) \mathrm{d}^{2} \boldsymbol{\rho} .
\end{aligned}
$$

We substitute here $\psi=\psi_{0}+\psi_{1}$, where $\psi_{1}$ is a small perturbation satisfying equations (6) and (8) in the vicinity of the defect and decaying to zero on a scale $\rho \sim \xi$ (if we use the function $\psi_{1}$ satisfying (6) in the whole $x y$ plane, the component $\Delta F_{2}$ will diverge to positive infinity). Close to the cavity the characteristic length scale for $\psi_{1}$ is $D \ll \xi$, hence, we can neglect terms of the order of $\left|\psi_{1}\right|^{2}$ as compared to the term $\xi^{2}\left|\nabla \psi_{1}\right|^{2}$ :

$$
\begin{aligned}
\Delta F_{2}= & \frac{\xi^{2} H_{\mathrm{c}}^{2}}{4 \pi n_{0}}\left\{\int_{\boldsymbol{\rho} \notin S}\left|\nabla \psi_{1}\right|^{2} \mathrm{~d}^{2} \boldsymbol{\rho}\right. \\
& \left.-\int_{\partial S}\left[\psi_{1}^{*}\left(\nabla \psi_{0} \boldsymbol{n}\right)+\psi_{1}\left(\nabla \psi_{0}^{*} \boldsymbol{n}\right)\right] \mathrm{d} \ell\right\} .
\end{aligned}
$$

Using equation (8) and applying the Gauss theorem, we transform the right-hand side of the last relation as follows:

$$
\begin{aligned}
\Delta F_{2} & =-\frac{\xi^{2} H_{\mathrm{c}}^{2}}{4 \pi n_{0}} \int_{\boldsymbol{\rho} \notin S}\left(\left|\nabla \psi_{1}\right|^{2}+\psi_{1}^{*} \nabla^{2} \psi_{1}\right. \\
& \left.+\psi_{1} \nabla^{2} \psi_{1}^{*}\right) \mathrm{d}^{2} \boldsymbol{\rho} .
\end{aligned}
$$

From (6) we find that

$$
\left|\psi_{1} \nabla^{2} \psi_{1}\right| \sim\left|\psi_{1}\right|^{2} / \xi^{2} \ll\left|\nabla \psi_{1}\right|^{2}
$$

hence

$$
\Delta F_{2} \approx-\frac{\xi^{2} H_{\mathrm{c}}^{2}}{4 \pi n_{0}} \int_{\boldsymbol{\rho} \notin S}\left|\nabla \psi_{1}\right|^{2} \mathrm{~d}^{2} \boldsymbol{\rho} .
$$

An explicit expression for $\Delta F_{2}$ in terms of $\psi_{0}$ will be given below (see (25)).

\subsection{Force balance equation}

In this section we derive the solvability condition for the system (6)-(8). Our derivation closely follows the computations from [21] which were used to determine the viscous drag force acting on a moving vortex.

First, we introduce the auxiliary function $\psi_{\mathrm{d}}=\boldsymbol{d} \nabla \psi_{0}$, where $\boldsymbol{d}$ is an arbitrary constant unit vector. $\psi_{\mathrm{d}}$ satisfies the equation

$$
-\xi^{2} \nabla^{2} \psi_{\mathrm{d}}-\psi_{\mathrm{d}}+2 n_{0}^{-1}\left|\psi_{0}\right|^{2} \psi_{\mathrm{d}}+n_{0}^{-1} \psi_{0}^{2} \psi_{\mathrm{d}}^{*}=0 .
$$

Let us multiply (6) by $\psi_{\mathrm{d}}^{*}$ and subtract (12) multiplied by $\psi_{1}^{*}$ from it. When we add the complex conjugate to the resulting equation we obtain

$$
\operatorname{div}\left(-\psi_{\mathrm{d}}^{*} \nabla \psi_{1}+\psi_{1} \nabla \psi_{\mathrm{d}}^{*}-\psi_{\mathrm{d}} \nabla \psi_{1}^{*}+\psi_{1}^{*} \nabla \psi_{\mathrm{d}}\right)=0 .
$$

We integrate the last relation over the region $\boldsymbol{\rho} \notin S,|\boldsymbol{\rho}-\boldsymbol{L}|<R$ and apply the Gauss theorem:

$$
\begin{aligned}
& \int_{|\boldsymbol{\rho}-\boldsymbol{L}|=R}\left(-\psi_{\mathrm{d}}^{*} \nabla \psi_{1}+\psi_{1} \nabla \psi_{\mathrm{d}}^{*}-\psi_{\mathrm{d}} \nabla \psi_{1}^{*}+\psi_{1}^{*} \nabla \psi_{\mathrm{d}}\right) \boldsymbol{n}_{1} \mathrm{~d} \ell \\
& -\int_{\partial S}\left(-\psi_{\mathrm{d}}^{*} \nabla \psi_{1}+\psi_{1} \nabla \psi_{\mathrm{d}}^{*}\right. \\
& \left.-\psi_{\mathrm{d}} \nabla \psi_{1}^{*}+\psi_{1}^{*} \nabla \psi_{\mathrm{d}}\right) \boldsymbol{n} \mathrm{d} \ell=0
\end{aligned}
$$

where $\boldsymbol{n}_{1}$ is the outward unit normal to the circle $|\boldsymbol{\rho}-\boldsymbol{L}|=R$. The first integral can be calculated with the help of (7):

$$
\begin{aligned}
& \int_{|\boldsymbol{\rho}-\boldsymbol{L}|=R}\left(-\psi_{\mathrm{d}}^{*} \nabla \psi_{1}+\psi_{1} \nabla \psi_{\mathrm{d}}^{*}-\psi_{\mathrm{d}} \nabla \psi_{1}^{*}+\psi_{1}^{*} \nabla \psi_{\mathrm{d}}\right) \boldsymbol{n} \mathrm{d} \ell \\
& \approx-\frac{2 \pi m}{e \hbar}\left[\boldsymbol{d} \cdot\left(\boldsymbol{z}_{0} \times \boldsymbol{j}_{\mathrm{tr}}\right)\right],
\end{aligned}
$$

where $z_{0}$ is the unit vector along the $z$ axis. Here we neglected terms of the order of $\xi^{2} / R^{2}$, which appear due to variations of the order parameter modulus.

The second integral in equation (13) can be transformed using (8) and the Gauss theorem:

$$
\begin{aligned}
\int_{\partial S}( & \left.-\psi_{\mathrm{d}}^{*} \nabla \psi_{1}-\psi_{\mathrm{d}} \nabla \psi_{1}^{*}+\psi_{1} \nabla \psi_{\mathrm{d}}^{*}+\psi_{1}^{*} \nabla \psi_{\mathrm{d}}\right) \boldsymbol{n} \mathrm{d} \ell \\
& \left.\approx S \cdot \operatorname{div}\left(\psi_{\mathrm{d}}^{*} \nabla \psi_{0}+\psi_{\mathrm{d}} \nabla \psi_{0}^{*}\right)\right|_{\boldsymbol{\rho}=0} \\
& +\int_{\partial S}\left(\psi_{1} \nabla \psi_{\mathrm{d}}^{*}(0)+\psi_{1}^{*} \nabla \psi_{\mathrm{d}}(0)\right) \boldsymbol{n} \mathrm{d} \ell .
\end{aligned}
$$

Here and further on we neglect terms which are much smaller than $n_{0} D^{2} / \xi^{3}$.

In order to proceed we have to determine the value of $\psi_{1}$ at the defect boundary. It has been noted in section 2.2 that $\psi_{1} \sim \sqrt{n_{0}} D \xi^{-1}$. Hence,

$$
\partial^{2} \psi_{1} / \partial x^{2} \sim \partial^{2} \psi_{1} / \partial y^{2} \sim \sqrt{n_{0}} D^{-1} \xi^{-1} \gg\left|\psi_{1}\right| \xi^{-2} .
$$

This implies that near the defect we can use the Laplace equation

$$
\nabla^{2} \psi_{1}=0
$$

instead of (6). The boundary condition can also be simplified:

$$
\left.\nabla \psi_{1} \boldsymbol{n}\right|_{\partial S}=-\nabla \psi_{0}(0) \boldsymbol{n} .
$$

Such a simplification is acceptable since we are not interested in small corrections of the order of $\sqrt{n_{0}} D^{2} / \xi^{2}$ to $\psi_{1}$. Equations (16) and (17) are equivalent to an electrostatic problem where $\psi_{1}$ plays the role of the electric potential of a charged cylinder. Note that these equations cannot be derived within the electrostatic approximation for the London theory [10], where variations of the superconducting phase are taken into account, but the order parameter modulus is assumed to be constant.

The relation

$$
\oint_{\partial S} \nabla \psi_{1} n \mathrm{~d} \ell=0
$$


can be interpreted as a vanishing total 'charge' of the cylinder. It provides that a solution of equations (16) and (17) exists that decays like $\rho^{-1}$ at infinity. This solution, which we denote as $\psi_{1}^{(d)}$, represents the irregular part of $\psi_{1}$ : it has singularities inside the defect. We define the regular component of $\psi_{1}$ as

$$
\psi_{1}^{(\mathrm{i})}=\psi_{1}-\psi_{1}^{(\mathrm{d})} .
$$

It is proved in the appendix that the contribution of $\psi_{1}^{(\mathrm{i})}$ to the integral in the right-hand side of equation (15) is negligible.

Combining (13)-(15) and using the fact that $\psi_{0}$ satisfies (1) we obtain

$$
\begin{aligned}
& \frac{2 \pi \xi^{2} m}{e \hbar}\left[\boldsymbol{d} \cdot\left(z_{0} \times \boldsymbol{j}_{\mathrm{tr}}\right)\right] \\
& \quad+\left.S(\boldsymbol{d} \nabla)\left(\xi^{2}\left|\nabla \psi_{0}\right|^{2}-\left|\psi_{0}\right|^{2}+\frac{\left|\psi_{0}\right|^{4}}{2 n_{0}}\right)\right|_{\boldsymbol{\rho}=0} \\
& \quad+\xi^{2} \int_{\partial S}\left(\psi_{1}^{(\mathrm{d})} \nabla \psi_{\mathrm{d}}^{*}(0)+\psi_{1}^{(\mathrm{d}) *} \nabla \psi_{\mathrm{d}}(0)\right) \boldsymbol{n} \mathrm{d} \ell \approx 0 .
\end{aligned}
$$

Owing to the linearity of equations (16) and (17) the solution can be presented in the form

$$
\psi_{1}^{(\mathrm{d})}=\left(\boldsymbol{g}(\boldsymbol{\rho}) ; \nabla \psi_{0}(0)\right)
$$

where $g$ is a real vector field defined by the relations

$$
\nabla^{2} \boldsymbol{g}=0,\left.\quad(\boldsymbol{n} \nabla) \boldsymbol{g}\right|_{\partial S}=-\boldsymbol{n},\left.\quad \boldsymbol{g}\right|_{\rho \rightarrow \infty}=0 .
$$

Then

$$
\begin{gathered}
\int_{\partial S}\left(\psi_{1}^{(\mathrm{d})} \nabla \psi_{\mathrm{d}}^{*}(0)+\psi_{1}^{(\mathrm{d}) *} \nabla \psi_{\mathrm{d}}(0)\right) \boldsymbol{n} \mathrm{d} \ell \\
=\left.(\boldsymbol{d} \nabla)\left(\nabla \psi_{0} \hat{\boldsymbol{G}} \nabla \psi_{0}^{*}\right)\right|_{\boldsymbol{\rho}=0},
\end{gathered}
$$

where $\hat{G}$ is a real symmetric matrix with components

$$
G_{i j}=\int_{\partial S} g_{i} n_{j} \mathrm{~d} \ell=\int_{\boldsymbol{\rho} \notin S} \nabla g_{i} \nabla g_{j} \mathrm{~d}^{2} \boldsymbol{\rho} .
$$

Equation (19) transforms into

$$
-\frac{\phi_{0}}{c}\left[\boldsymbol{d} \cdot\left(z_{0} \times \boldsymbol{j}_{\mathrm{tr}}\right)\right]-\left(\boldsymbol{d} \nabla_{\boldsymbol{L}}\right) U_{\mathrm{p}}=0 .
$$

Here $\phi_{0}=\pi \hbar c / e$ is the flux quantum, $\nabla_{\boldsymbol{L}}=\partial / \partial \boldsymbol{L}$, and

$$
\begin{aligned}
U_{\mathrm{p}}= & -\left.S \frac{H_{\mathrm{c}}^{2}}{4 \pi n_{0}}\left(\xi^{2}\left|\nabla \psi_{0}\right|^{2}-\left|\psi_{0}\right|^{2}+\frac{\left|\psi_{0}\right|^{4}}{2 n_{0}}\right)\right|_{\rho=0} \\
& -\xi^{2} \frac{H_{\mathrm{c}}^{2}}{4 \pi n_{0}} \nabla \psi_{0}(0) \hat{G} \nabla \psi_{0}^{*}(0) .
\end{aligned}
$$

Since $\boldsymbol{d}$ is an arbitrary vector, it can be dropped, and we finally obtain the force balance equation, connecting the vortex displacement $\boldsymbol{L}$ with the transport current $\boldsymbol{j}_{\mathrm{tr}}$ :

$$
-\frac{\phi_{0}}{c}\left(z_{0} \times j_{\text {tr }}\right)-\nabla_{L} U_{\mathrm{p}}=0 .
$$

Here, the first term is the Lorentz force and the second term is the pinning force: $\boldsymbol{F}_{\mathrm{p}}=-\nabla_{\boldsymbol{L}} U_{\mathrm{p}}$. Thus, we may conclude that $U_{\mathrm{p}}$ is the pinning potential. It can be proved that this definition of the pinning potential is identical to the one given in section 2.2, if $\psi_{1}$ in equation (11) is replaced by $\psi_{1}^{(\mathrm{d})}$. Thus,

$$
\Delta F_{2}=-\xi^{2} \frac{H_{\mathrm{c}}^{2}}{4 \pi n_{0}} \nabla \psi_{0}(0) \hat{G} \nabla \psi_{0}^{*}(0)
$$

Before we determine some pinning potentials explicitly, we would like to note that our consideration can be easily generalized for the anisotropic case. Indeed, generally, the GL free energy can be presented in the form

$$
\begin{aligned}
F= & \frac{H_{\mathrm{c}}^{2}}{4 \pi n_{0}} \int\left(\xi_{x}^{2}\left|\frac{\partial \psi}{\partial x}\right|^{2}+\xi_{y}^{2}\left|\frac{\partial \psi}{\partial y}\right|^{2}+\xi_{z}^{2}\left|\frac{\partial \psi}{\partial z}\right|^{2}\right. \\
& \left.-|\psi|^{2}+\frac{|\psi|^{4}}{2 n_{0}}\right) d^{3} \boldsymbol{r},
\end{aligned}
$$

where $\xi_{x}, \xi_{y}$ and $\xi_{z}$ are the coherence lengths for different directions. The scaling transformation $\tilde{x}=x, \tilde{y}=y \xi_{x} / \xi_{y}$, and $\tilde{z}=z \xi_{x} / \xi_{z}$ reduces the free energy to the isotropic form. Thus, we again arrive at equations (1)-(3).

Now we consider two types of defects.

\subsection{A circular defect}

Let the defect be a circular cylinder with the radius $a$. When the origin is placed on the axis of the cylinder, the decaying solution of equations (16) and (17) is

$$
\psi_{1}^{(\mathrm{d})}=\frac{a^{2}\left(\nabla \psi_{0} \cdot \boldsymbol{\rho}\right)}{\rho^{2}},
$$

and the pinning potential is

$$
U_{\mathrm{p}}(\boldsymbol{L})=-\left.\frac{H_{\mathrm{c}}^{2} a^{2}}{4 n_{0}}\left(2 \xi^{2}\left|\nabla \psi_{0}\right|^{2}-\left|\psi_{0}\right|^{2}+\frac{\left|\psi_{0}\right|^{4}}{2 n_{0}}\right)\right|_{\boldsymbol{\rho}=0} .
$$

The function $\psi_{0}$ can be determined numerically from the GL equation (6). We are not going into details of these calculations here. A detailed numerical analysis of this function can be found in [22]. Of course, the pinning potential is proportional to the defect volume, as has been noted in [6]

Equation (28) allows us to determine the pinning energy, $E_{\mathrm{p}}$, and to compare it with a numerical result from a preceding paper. According to our calculations,

$$
E_{\mathrm{p}}=U_{\mathrm{p}}(\infty)-U_{\mathrm{p}}(0)=0.47 H_{\mathrm{c}}^{2} a^{2},
$$

which coincides with the numerical value given in [13] up to a factor of the order of unity.

The profiles of the pinning potential and the pinning force are plotted in figure 2. The pinning force reaches its maximum at $L=L_{\mathrm{cr}}=0.84 \xi$, where $F_{\mathrm{p}}=F_{\mathrm{cr}}=0.252 H_{\mathrm{c}}^{2} a^{2} / \xi$. When $j_{\text {tr }}>c F_{\mathrm{cr}} /\left|\phi_{0}\right|$, the force balance equation (24) has no solutions, hence

$$
j_{\mathrm{d}}=c F_{\mathrm{cr}} /\left|\phi_{0}\right|=0.252 \frac{H_{\mathrm{c}}^{2} a^{2}|e|}{\pi \hbar \xi}
$$

is the depinning current. When $j<j_{\mathrm{d}}$ equation (24) has two solutions due to the nonmonotonic behavior of the function $F_{\mathrm{p}}(L)$, but the solution with the larger vortex displacement is 

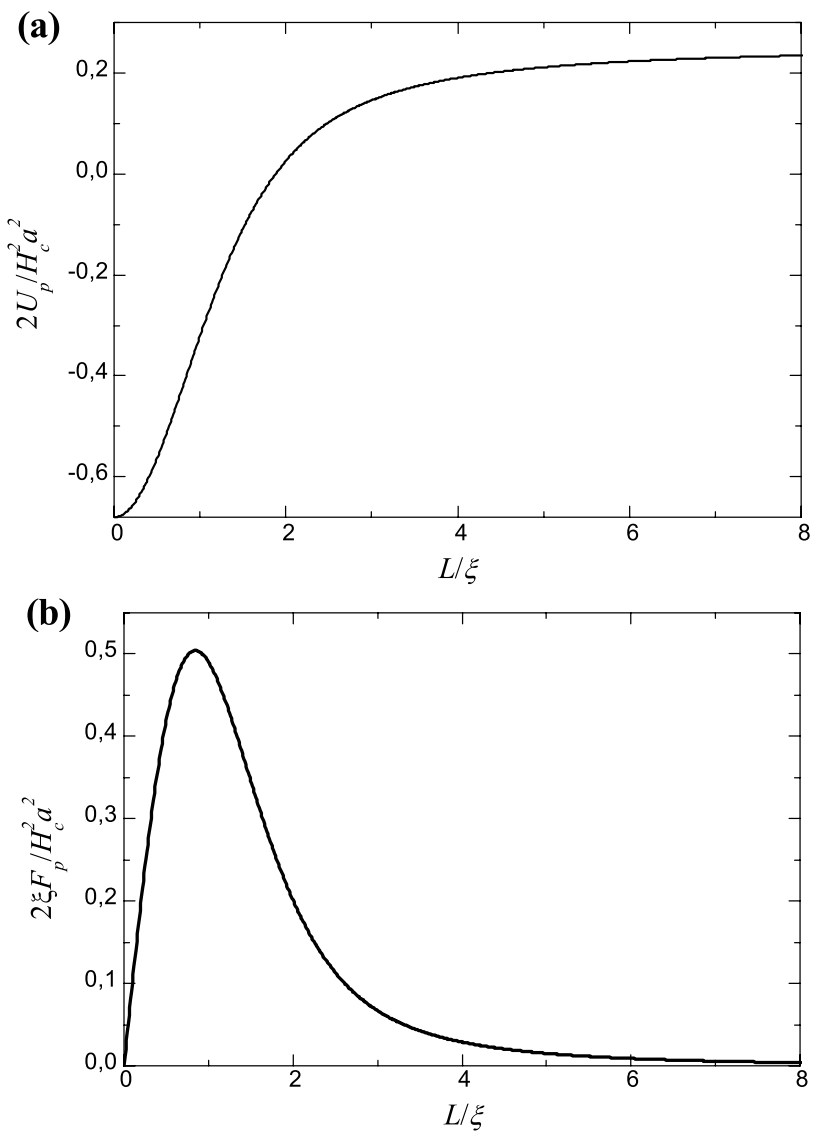

Figure 2. Profiles of the pinning potential (a) and the pinning force (b) for a circular defect.

thermodynamically unstable. Indeed, it can be easily proved that it corresponds to a saddle point of the correction $\Delta F$ to the free energy of a vortex connected with the presence of the defect and the transport current:

$$
\Delta F=U_{\mathrm{p}}+\frac{\phi_{0}}{c}\left[\left(z_{0} \times \boldsymbol{j}_{\mathrm{tr}}\right) \boldsymbol{L}\right] .
$$

Certainly, the depinning current, given by (29), is much smaller than the depairing current

$$
J_{\mathrm{c}}=\frac{2|e| \xi H_{\mathrm{c}}^{2}}{3 \sqrt{3} \pi \hbar},
$$

hence, it cannot destroy the superconducting order parameter in the sample.

Equation (29) predicts that the depinning current grows like $a^{2}$ when the defect size is increased. Obviously, this quadratic growth rate must slow down when the defect size is of the order of the coherence length (otherwise, the depinning current would eventually exceed $J_{\mathrm{c}}$ ). Thus, for $a \sim \xi$ equation (29) should give an upper estimate for $j_{\mathrm{d}}$. This argument is well confirmed by the fact that the numerical value of the depinning current for the defect radius $0.25 \xi$,

$$
j_{\mathrm{d}}=0.01 \frac{H_{\mathrm{c}}^{2} \xi|e|}{\pi \hbar}
$$

given in [14], is 1.5 times smaller than our estimate.
To sum up, depinning from a circular defect occurs as follows: as the transport current increases from zero to $j_{\mathrm{d}}$ the vortex displacement with respect to the origin increases from zero to $L_{\mathrm{cr}}$; as the current is increased further the vortex is carried away from the pinning cite. Thus, in some range of currents below $j_{\mathrm{d}}$ a stable bound state exists, where the vortex center is situated outside the defect. However, in [14] this state has not been detected numerically. This disagreement with our result may be due to the relatively large size of defects considered in the paper $(a \geq 0.25 \xi)$, and also due to the strong influence of the outer periphery of the cell where the GL equation has been integrated.

Finally, we would like to stress that the expression (28) for the single-vortex pinning potential does not depend on whether the vortex interacts with other vortices or not (the only restriction is that the vortex cores must not overlap). Of course, the given above expression (29) for the critical current can be applied directly only provided that all vortices are pinned, and the vortex-vortex interaction force can be neglected as compared to the pinning force. This may be achieved if the applied magnetic field is close to the first critical field. Still, even if the above conditions are violated, the expression (28) for the pinning potential will be useful for the estimation of the critical current, if the interaction between vortices is properly taken into account (see [5]).

\subsection{An elliptical defect}

Consider an elliptical defect with the cross-section

$$
\frac{x^{2}}{a^{2}}+\frac{y^{2}}{b^{2}}<1
$$

where $a>b$. We shall determine the vector field $\boldsymbol{g}$ and the pinning potential. It is convenient to use the elliptical coordinates $(\zeta, \eta)$ :

$x=\sqrt{a^{2}-b^{2}} \cosh \zeta \cos \eta, \quad y=\sqrt{a^{2}-b^{2}} \sinh \zeta \sin \eta$.

The border of the defect corresponds to the value $\zeta=\zeta_{0}$, where

$$
\sinh \zeta_{0}=\frac{b}{\sqrt{a^{2}-b^{2}}}, \quad \cosh \zeta_{0}=\frac{a}{\sqrt{a^{2}-b^{2}}} .
$$

Equations (21) in the new coordinates read

$$
\begin{aligned}
\frac{\partial^{2} \boldsymbol{g}}{\partial \zeta^{2}}+\frac{\partial^{2} \boldsymbol{g}}{\partial \eta^{2}}=0, & \left.\boldsymbol{g}\right|_{\zeta \rightarrow \infty} & =0 \\
\left.\frac{\partial g_{x}}{\partial \zeta}\right|_{\zeta=\zeta_{0}}=-b \cos \eta, & \left.\frac{\partial g_{y}}{\partial \zeta}\right|_{\zeta=\zeta_{0}} & =-a \sin \eta .
\end{aligned}
$$

The solution is

$$
g_{x}=b \mathrm{e}^{\zeta_{0}-\zeta} \cos \eta, \quad g_{y}=a \mathrm{e}^{\zeta_{0}-\zeta} \sin \eta .
$$

Using (22), we obtain the components of the matrix $\hat{G}$ :

$$
\begin{gathered}
G_{x x}=\pi b^{2}, \quad G_{y y}=\pi a^{2}, \\
G_{x y}=G_{y x}=0 .
\end{gathered}
$$


Hence, according to equation (23), the pinning potential is

$$
\begin{aligned}
U_{\mathrm{p}}= & -\frac{H_{\mathrm{c}}^{2}}{4 n_{0}}\left[a b\left(\xi^{2}\left|\nabla \psi_{0}\right|^{2}-\left|\psi_{0}\right|^{2}+\frac{\left|\psi_{0}\right|^{4}}{2 n_{0}}\right)\right. \\
& \left.+\xi^{2} b^{2}\left|\frac{\partial \psi_{0}}{\partial x}\right|^{2}+\xi^{2} a^{2}\left|\frac{\partial \psi_{0}}{\partial y}\right|^{2}\right]\left.\right|_{\rho=0} \cdot
\end{aligned}
$$

The potential well for the vortex now does not have cylindrical symmetry. As a result, the vortex displacement $L$ and the depinning threshold $j_{\mathrm{d}}$ depend on the direction of the transport current.

\section{Comparison with the London theory}

In a preceding paper [11] the pinning potential in the presence of a circular and elliptical cavity has been derived within the London theory. The applicability condition for this approach is that the defect size be much larger than the temperature-dependent coherence length, i.e., $D \gg \xi(T)$, so it may seem that the results from [11] cannot be compared with ours. However, the condition $D \ll \xi$ is not essential for our calculations. Indeed, instead we may demand (i) $\left|\psi_{1}\right| \ll \sqrt{n_{0}}$ and (ii): the quantity $\nabla \psi_{0}$ should be approximately constant in the area occupied by the cavity. These two conditions are satisfied when

$$
D \gg \xi \quad \text { and } \quad L \gg D,
$$

so for a large defect and large vortex-defect distance our pinning potential should coincide with the one obtained within the London theory.

For a circular defect our pinning potential (28) at large vortex-defect distances, $L \gg a$, equals

$$
U_{\mathrm{p}}=-\left(\frac{\phi_{0}}{4 \pi \lambda}\right)^{2} \frac{a^{2}}{L^{2}}+\text { const. }
$$

This expression is in good agreement with equation (5) from [11]. For an elliptical hole equation (35) yields in the $L \gg a$ limit

$$
U_{\mathrm{p}}=-\left(\frac{\phi_{0}}{4 \pi \lambda}\right)^{2} \frac{1}{2}\left(\frac{a b}{L^{2}}+\frac{b^{2} L_{y}^{2}}{L^{4}}+\frac{a^{2} L_{x}^{2}}{L^{4}}\right)+\text { const },
$$

whereas the potential from [11] is

$$
U_{\mathrm{p}}=-\left(\frac{\phi_{0}}{4 \pi \lambda}\right)^{2}\left(\frac{a+b}{2}\right)^{2} \frac{1}{L^{2}},
$$

which, obviously, does not coincide with (38). Below we will explain the reason for this discrepancy.

The derivation of the interaction energy between a vortex and a cavity in the London approximation is based on the equation

$$
U_{\mathrm{p}}=\frac{\phi_{0} h_{\mathrm{im}}(\boldsymbol{L})}{8 \pi},
$$

where $h_{\mathrm{im}}$ is the $z$ projection of the field created by image vortices. This field can be expressed as

$$
h_{\mathrm{im}}=h_{z}-\frac{\phi_{0}}{2 \pi \lambda^{2}} \ln \left|\frac{\lambda^{2}}{\zeta-\zeta_{0}}\right|,
$$

where $\zeta=x+\mathrm{i} y, \zeta_{0}=L_{x}+\mathrm{i} L_{y}$, and $h_{z}$ is the full magnetic field, satisfying the Poisson equation

$$
\nabla^{2} h_{z}=-\frac{\phi_{0}}{\lambda^{2}} \delta(\boldsymbol{\rho}-\boldsymbol{L}) .
$$

The second term on the right-hand side of equation (41) represents the self-field of the vortex with the opposite sign. For a circular defect with a radius $a_{0}$, the image field at the position of the vortex is

$$
h_{\mathrm{im}}^{\mathrm{c}}\left(\zeta_{0}\right)=\frac{\phi_{0}}{2 \pi \lambda^{2}} \ln \left(1-\frac{a_{0}^{2}}{\left|\zeta_{0}\right|^{2}}\right) .
$$

In order to obtain the magnetic field in the presence of a non-circular defect, we may apply a conformal transformation $w=w(\zeta)$ to the $\zeta$ plane. Since the form of Poisson's equation is not modified by such a transformation, the field distribution in the $w$ plane is given by

$$
h_{z}(w)=h_{z}^{\mathrm{c}}(\zeta(w))
$$

where $h_{z}^{\mathrm{c}}(\zeta)$ is the solution of equation (42) in the presence of a circular defect. Using the definition (41) of the image field, we obtain

$$
\begin{aligned}
h_{\mathrm{im}}\left(w_{0}\right)= & h_{\mathrm{im}}^{\mathrm{c}}\left(\zeta\left(w_{0}\right)\right)+\left[\frac{\phi_{0}}{2 \pi \lambda^{2}} \ln \left|\frac{\lambda}{\zeta(w)-\zeta_{0}}\right|\right. \\
& \left.-\frac{\phi_{0}}{2 \pi \lambda^{2}} \ln \left|\frac{\lambda}{w-w_{0}}\right|\right]\left.\right|_{w=w_{0}},
\end{aligned}
$$

where $w_{0}=w\left(\zeta_{0}\right)$ specifies the position of the vortex in the $w$-plane. Hence, the pinning potential equals

$$
U_{\mathrm{p}}=\left(\frac{\phi_{0}}{4 \pi \lambda}\right)^{2}\left[\ln \left(1-\frac{R^{2}}{\left|\zeta\left(w_{0}\right)\right|^{2}}\right)-\ln \left|\frac{\mathrm{d} \zeta}{d w}\left(w_{0}\right)\right|\right] .
$$

Here, the first logarithmic term originates from the transformation of the image field (43), while the second term is connected with the modification of the self-field of the vortex. In [11] this term has not been taken into account. As a result, the isotropic potential (39) has been obtained. In order to determine the correct pinning potential for an elliptical cavity, we apply the modified Joukowski transformation [11]:

$$
\begin{aligned}
w(\zeta) & =\frac{a+b}{2} \frac{\zeta}{a_{0}}+\frac{a-b}{2} \frac{a_{0}}{\zeta} ; \\
U_{\mathrm{p}} & =\left(\frac{\phi_{0}}{4 \pi \lambda}\right)^{2}\left[\ln \left(1-\left|\frac{a+b}{w+\sqrt{w^{2}-a^{2}+b^{2}}}\right|^{2}\right)\right. \\
& \left.-\ln \left|1+\frac{w}{\sqrt{w^{2}-a^{2}+b^{2}}}\right|\right]+ \text { const. }
\end{aligned}
$$

For $|w| \gg a$ this expression coincides with our result obtained within the GL theory (see (38)).

\section{Conclusion}

By solving the Ginzburg-Landau equation, we developed a method to determine analytically the pinning potential for a vortex interacting with a small cylindrical cavity. This method 
has been applied to a circular and elliptical defect. In the latter case, the pinning potential appeared to be anisotropic, as one would expect. Also, we recalculated the pinning potential for an elliptical cavity within the London theory, using the conformal transformation technique [11], considering the modification of the image field as well as the transformation of the self-field of the vortex, which had not been previously taken into account. Our results obtained within the GL and London theories agree well with each other in the range of parameters, where both approaches are valid.

All our previous consideration has been related to the case of a vortex strictly parallel to the defect. This assumption is obviously satisfied in thin superconducting films, where the transport current is distributed almost uniformly over the film thickness. However, it has been claimed [23] that in a bulk superconductor depinning is likely to occur due to vortex kink formation in a surface layer with the thickness equal to the London length. Still, if the vortex radius of curvature is large as compared to $\xi$, and the vortex axis makes a small angle with the defect axis, our approach should give reasonable estimates of the pinning energy and of the local vortex displacement with respect to the defect. Moreover, in the presence of a sufficiently small transport current a bound state should occur, when the vortex core is outside the defect (at least in a surface layer-in bulk superconductors). This bound state should be observable, for example, using scanning tunneling microscopy.

Our results are closely related to recent experimental studies of the vortex ratchet effect [24, 25], where an anisotropic pinning structure is a key element. Note that the pinning centers and experimental conditions in [25] almost perfectly match our model: the antidots embedded into the thin Al films represent insulating inclusions, and the typical temperatures are very close to the critical temperature, so that the coherence length is larger than the defect size. Thus, our expressions for the pinning potential may be useful for vortex ratchet design and for the interpretation of future experiments in this field.

\section{Acknowledgments}

We are grateful to A I Buzdin for helpful discussions. This work was supported, in part, by European IRSES program SIMTECH (contract no. 246937), the Russian Foundation for Basic Research, FTP Scientific and Educational Personnel of Innovative Russia in 2009-13, and the program of LEA 'Physique Théorique et Matière Condensée'.

\section{Appendix}

In this appendix we will demonstrate that the contribution from the function $\psi_{1}^{(i)}$ (see (18)) to the pinning force is negligible. It is sufficient to prove that the absolute value of the integral

$$
I=\int_{\partial S}\left(\psi_{1}^{(\mathrm{i})} \nabla \psi_{\mathrm{d}}^{*}(0)+\psi_{1}^{(\mathrm{i}) *} \nabla \psi_{\mathrm{d}}(0)\right) \boldsymbol{n} \mathrm{d} \ell
$$

is much smaller than $n_{0} D^{2} / \xi^{3}$.
The function $\psi_{1}^{(\mathrm{i})}$ has the following properties:

$$
\left.\nabla \psi_{1}^{(\mathrm{i})} \boldsymbol{n}\right|_{\partial S} \approx 0, \quad \nabla^{2} \psi_{1}^{(\mathrm{i})}=0, \quad \rho<r,
$$

where $r$ is a quantity of the order of the coherence length. Let us introduce an auxiliary function $v$ defined by the relations

$$
\nabla^{2} v=0,\left.\quad \boldsymbol{n} \nabla v\right|_{\partial S}=\xi \boldsymbol{n} \nabla \psi_{\mathrm{d}}^{*}(0),\left.\quad v\right|_{\rho \rightarrow \infty}=0 .
$$

The properties of this function are identical to those of $\psi_{1}^{(\mathrm{d})}$ : it is of the order of $\sqrt{n_{0}} D \xi^{-1}$ at the defect border and decays like $\rho^{-1}$ at infinity. For a smooth defect $v \sim \sqrt{n_{0}} D^{2} / \xi \rho$. Now we make some simple calculations:

$$
\begin{aligned}
0= & \int_{\rho \notin S, \rho<r}\left(\psi_{1}^{(\mathrm{i})} \nabla^{2} v-v \nabla^{2} \psi_{1}^{(\mathrm{i})}\right) \mathrm{d}^{2} \boldsymbol{\rho} \\
& =-\int_{\partial S} \psi_{1}^{(\mathrm{i})} \nabla v \boldsymbol{n} \mathrm{d} \ell+\int_{\rho=r}\left(\psi_{1}^{(\mathrm{i})} \frac{\partial v}{\partial \rho}-v \frac{\partial \psi_{1}^{(\mathrm{i})}}{\partial \rho}\right) \mathrm{d} \ell .
\end{aligned}
$$

Since $\psi_{1}^{(\mathrm{i})} \approx \psi_{1}$ when $\rho \sim r$,

$$
I=\xi^{-1} \int_{\rho=r}\left(\psi_{1} \frac{\partial v}{\partial \rho}-v \frac{\partial \psi_{1}}{\partial \rho}\right) \mathrm{d} \ell+\text { c.c. },
$$

where c.c. denotes the complex conjugate. According to statement (B) from section 2.1, when $\rho=r,\left|\psi_{1}\right| \ll \sqrt{n_{0}}$ and $\left|\partial \psi_{1} / \partial \rho\right| \ll \sqrt{n_{0}} / \xi$, since the characteristic length scale is $\xi$, then it follows immediately from (49) that $|I| \ll n_{0} D^{2} / \xi^{3}$.

\section{References}

[1] Civale L, Marwick A D, Worthington T K, Kirk M A, Thompson J R, Krusin-Elbaum L, Sun Y, Clem J R and Holtzberg F 1991 Phys. Rev. Lett. 67648

[2] Goyal A et al 2005 Supercond. Sci. Technol. 181533

[3] Solovyov V F, Li Q, Si W, Maiorov B, Haugan T J, MacManus-Driscoll J L, Yao H, Jia Q X and Specht E D 2012 Phys. Rev. B 86094511

Maiorov B, Katase T, Usov I O, Weigand M, Civale L, Hiramatsu H and Hosono H 2012 Phys. Rev. B 86094513

Xu A, Braccini V, Jaroszynski J, Xin Y and Larbalestier D C 2012 Phys. Rev. B 86115416

Haberkorn N et al 2012 Phys. Rev. B 85174504

[4] Welp U, Xiao Z L, Jiang J S, Vlasko-Vlasov V K, Bader S D, Crabtree G W, Liang J, Chik H and Xu J M 2002 Phys. Rev. B 66212507

Raedts S, Silhanek A V, Van Bael M J and Moshchalkov V V 2004 Phys. Rev. B 70024509

Karapetrov G, Fedor J, Iavarone M, Rosenmann D and Kwok W K 2005 Phys. Rev. Lett. 95167002

[5] Blatter G, Feigel'man M V, Geshkenbein V B, Larkin A I and Vinokur V M 1994 Rev. Mod. Phys. 661125

[6] Campbell A M and Evetts J E 1972 Adv. Phys. 21199

[7] Mkrtchyan G S and Schmidt V V 1971 Zh. Eksp. Teor. Fiz. 61367 (in Russian)

Mkrtchyan G S and Schmidt V V 1972 Sov. Phys._JETP 34195 (Engl. transl.)

[8] Khalfin I B and Shapiro B Ya 1993 Physica C 207359

[9] Nordborg H and Vinokur V M 2000 Phys. Rev. B 6212408

[10] Buzdin A and Feinberg D 1996 Physica C 256303

[11] Buzdin A and Daumens M 1998 Physica C 294257

[12] Buzdin A and Daumens M 2000 Physica C 332108

[13] Maurer S M, Yeh N C and Tombrello T A 1996 Phys. Rev. B 5415372

[14] Maurer S M, Yeh N C and Tombrello T A 1998 J. Phys.: Condens. Matter 107429 
[15] Priour D J Jr and Fertig H A 2003 Phys. Rev. B 67054504

[16] Rosenstein B, Shapiro I and Shapiro B Ya 2010 Phys. Rev. B 81064507

[17] Rosenstein B, Shapiro I, Shapiro B Ya, Dingping Li, Frydman A, Poran S and Berco D 2012 Phys. Rev. B 85054512

[18] Thuneberg E V, Kurkijärvi J and Rainer D 1982 Phys. Rev. Lett. 481853

[19] Friesen M and Muzikar P 1996 Phys. Rev. B 53 R11953

[20] Mel'nikov A S, Samokhvalov A V and Zubarev M N 2009 Phys. Rev. B 79134529

Mel'nikov A S and Samokhvalov A V 2011 Pis. Zh. Eksp. Teor. Fiz. 94823 (in Russian)

Mel'nikov A S and Samokhvalov A V 2011 JETP Lett. 94759 (Engl. transl)

[21] Gor'kov L P and Kopnin N B 1975 Usp. Fiz. Nauk 116413 (in Russian)

Gor'kov L P and Kopnin N B 1975 Sov. Phys._Usp. 18496 (Engl. transl.)

[22] Hu C R 1972 Phys. Rev. B 61756
[23] Schuster Th, Indenbom M V, Kuhn H, Kronmüller H, Leghissa M and Kreiselmeyer G 1994 Phys. Rev. B 509499 Schuster Th, Kuhn H, Indenbom M, Leghissa M, Kraus M and Konczykowski M 1995 Phys. Rev. B 5116358

Schuster Th, Kuhn H, Indenbom M, Kreiselmeyer G, Leghissa M and Klaumünzer S 1996 Phys. Rev. B 532257

Indenbom M V, van der Beek C J, Konczykowski M and Holtzberg F 2000 Phys. Rev. Lett. 841792

[24] Villegas J E, Savel'ev S, Nori F, Gonzalez E M, Anguita J V, García R and Vicent J L 2003 Science 3021188

Wördenweber R, Dymashevski P and Misko V R 2004 Phys. Rev. B 69184504

Van de Vondel J, de Souza Silva C C, Zhu B Y, Morelle M and Moshchalkov V V 2005 Phys. Rev. Lett. 94057003

Wu T C, Horng L, Wu J C, Cao R, Koláček J and Yang T J 2007 J. Appl. Phys. 102033918

Jin B B, Zhu B Y, Wördenweber R, de Souza Silva C C, Wu P H and Moshchalkov V V 2010 Phys. Rev. B 81174505

[25] de Souza Silva C C, Van de Vondel J, Zhu B Y, Morelle M and Moshchalkov V V 2006 Phys. Rev. B 73014507 